ISSN 2072-6643

www.mdpi.com/journal/nutrients

Article

\title{
Sasa borealis Stem Extract Attenuates Hepatic Steatosis in High-Fat Diet-induced Obese Rats
}

\author{
Yuno Song ${ }^{1, \dagger}$, Soo-Jung Lee ${ }^{2, \dagger}$, Sun-Hee Jang ${ }^{1}$, Ji Hee Ha ${ }^{3}$, Young Min Song ${ }^{3}$, Yeoung-Gyu Ko ${ }^{4}$, \\ Hong-Duck Kim ${ }^{5}$, Wongi Min ${ }^{1}$, Suk Nam Kang ${ }^{6, *}$ and Jae-Hyeon Cho ${ }^{1, *}$
}

1 Institute of Agriculture and Life Science, College of Veterinary Medicine, Gyeongsang National University, Jinju 660-701, Korea; E-Mails: yunosong0805@gmail.com (Y.S.); sunhee5321@naver.com (S.-H.J.); wongimin@gnu.ac.kr (W.M.)

2 Department of Foods and Nutrition, Gyeongsang National University, Jinju 660-701, Korea; E-Mail: bodry96@hanmail.net

3 Department of Animal Science \& Biotechnology, Gyeongnam National University of Science and Technology, Jinju 660-758, Korea; E-Mails: hee71111@nate.com (J.H.H.); pigsong@gntech.ac.kr (Y.M.S.)

4 Animal Genetic Resources Station, National Institute of Animal Science, RDA, Namwon 590-832, Korea; E-Mail: kog4556@korea.kr

5 Department of Environmental Health Science, New York Medical College, Valhalla, NY 10595, USA; E-Mail: HongDuck_Kim@nymc.edu

6 Department of Bioindustry, Daegu University, Gyungsan 712-714, Korea

$\dagger$ These authors contributed equally to this work.

* Authors to whom correspondence should be addressed;

E-Mails:whitenightt@hanmail.net (S.N.K.); jaehcho@gnu.ac.kr (J.-H.C.);

Tel.: +82-53-850-6720 (S.N.K.); +82-55-772-2358 (J.H.C.);

Fax: +82-53-850-6729 (S.N.K.); +82-55-772-2349 (J.H.C.)

Received: 15 April 2014; in revised form: 12 May 2014 / Accepted: 26 May 2014 /

Published: 5 June 2014

\begin{abstract}
The aim of the current study is to examine the improving effect of Sasa borealis stem (SBS) extract extracts on high-fat diet (HFD)-induced hepatic steatosis in rats. To determine the hepatoprotective effect of SBS, we fed rats a normal regular diet (ND), HFD, and HFD supplemented with $150 \mathrm{mg} / \mathrm{kg}$ body weight (BW) SBS extracts for five weeks. We found that the body weight and liver weight of rats in the HFD + SBS group were
\end{abstract}


significantly lower than those in the HFD group. Significantly lower serum total cholesterol (TC) and triglyceride (TG) concentrations were observed in the SBS-supplemented group compared with the HFD group. We also found that the HFD supplemented with SBS group showed dramatically reduced hepatic lipid accumulation compared to the HFD alone group, and administration of SBS resulted in dramatic suppression of TG, TC in the HFD-induced fatty liver. In liver gene expression within the SBS treated group, PPAR $\alpha$ was significantly increased and SREBP-1c was significantly suppressed. SBS induced a significant decrease in the hepatic mRNA levels of PPAR $\gamma$, FAS, ACC1, and DGAT2. In conclusion, SBS improved cholesterol metabolism, decreased lipogenesis, and increased lipid oxidation in HFD-induced hepatic steatosis in rats, implying a potential application in treatment of non-alcoholic fatty liver disease.

Keywords: high-fat diet; Sasa borealis stem; hepatic steatosis; hepatic gene expression

\section{Introduction}

Obesity-related nonalcoholic fatty liver disease (NAFLD), one of the most common liver diseases, is significantly associated with metabolic syndrome, including obesity, dyslipidemia, and insulin resistance [1,2]. A hypercaloric dietary habit easily results in increased body weight, serum lipids, and hepatic lipid accumulation. Increased liver lipid accumulation causes lipid peroxidation, leading to further advancement of liver damage. Accumulation of triglycerides, termed as hepatic steatosis, which is characterized by fibrosis and necroinflammation, and can progress to cirrhosis and terminal liver failure, has been proposed as an indication of more severe liver disease [3]. Of particular importance, hepatic steatosis is always coupled with other diseases, i.e., obesity, diabetes, and hyperlipidemia [4]. Therefore, the clinical implications of hepatic steatosis are due mainly to its potential to cause chronic inflammation and then progress to cirrhosis and liver failure.

One of the major causes of lipid accumulation in NAFLD is the inability of the liver to regulate changes in lipogenesis in the transition from fasted to fed state [5]. Several studies have suggested that hepatic lipogenesis is increased in hepatic steatosis, which may result from either increased triglyceride synthesis, or decreased fatty acid oxidation through production of malony-CoA, both leading to increased triglyceride content in the liver [6]. Excess fat accumulation ultimately leads to development of hepatic steatosis and worsening hepatic insulin resistance via a network of transcription factors [7], which regulate hepatic lipogenesis and fatty acid oxidation, including sterol regulatory element-binding protein-1c (SREBP-1c), liver X receptor, and peroxisome proliferator receptors (PPARs). In addition, normalizing serum lipids is also known to be a way to hinder the occurrence of hepatic steatosis. The potential lipid lowering effect might be mediated by down-regulation of various lipogenic enzyme activities. Enzymes of the lipogenic pathway that are transcriptionally regulated include acetyl-CoA carboxylase (ACC), fatty-acid synthase (FAS), diacylglycerol acyltransferase2 (DGAT2), and stearoyl-CoA desaturase (SCD-1).

There has been a substantial effort toward understanding the mechanisms underlying NAFLD induced metabolic disorders and numerous studies have been conducted in the search for natural active 
products, particularly potential sources of antioxidant [8]. Bamboo has been used for medicinal purposes for centuries in Korea and other Asian countries, and its efficacy has been recorded in the materia medica, DongEuiBoGam in Korea [9,10]. Recent scientific research has demonstrated the health benefits of Bamboo leaves [10,11], culm [12], shoot [13], and shavings [14].

The medicinal effects of Sasa borealis, a species of Bamboo, are predominantly anti-diabetic through enhancement of insulin secretion [15], hypoglycemic and hypolipidemic effects [11], anti-obesity [16] and/or anti-oxidant effects [17]. In addition, clinical uses of Bamboo for treatment of hypertension, arteriosclerosis, cardiovascular disease, and cancer have been described [18].

Currently, no studies investigating the effects of dietary supplementation with Sasa borealis stem (SBS) extracts on fat metabolism in a high-fat diet (HFD)-induced NAFLD model have been reported. In the current study, we investigated the effects of SBS on lipid levels and cholesterol levels in the liver and serum of rats fed a HFD, and on expression of genes involved in fatty acid synthesis and oxidation in the liver.

\section{Experimental Section}

\subsection{Preparation of Sasa borealis Stem (SBS) Extracts}

Briefly, the stems of Sasa borealis were collected during the autumn season in Gyeongnam Province, Korea. The coarse powder of SBS was obtained after comminution and filtration (20-40 mesh) and $20 \mathrm{~g}$ powder were ground in an $80 \%(\mathrm{v} / \mathrm{v})$ methanol solution using a mixer, followed by extraction of the samples for three days with vigorous shaking. The filtrate was then isolated by membrane filtration for removal of macro- and micro-molecular components, such as polysaccharides and minerals. The extraction yield from dry weight of SBS was $19.6 \%$. The methanol extracts of the SBS were concentrated using rotary-vacuum evaporation at $50{ }^{\circ} \mathrm{C}$ and then freeze-dried.

\subsection{Measurement of Total Phenolic Content Using the Folin-Ciocalteu Assay}

The total phenolic content of the SBS was determined using a spectrophotometer according to the Folin-Ciocalteu colorimetric method [19]. Because gallic acid is one of the polyphenol compounds found in SBS, the total phenolic content of methanol extract of SBS was expressed as mg gallic acid (Sigma-Aldrich, USA) equivalents (GAE)/g.

\subsection{Measurement of Total Flavonoids}

The total flavonoid content was determined as previously described [20] with slight modifications. Briefly, $0.25 \mathrm{~mL}$ of SBS extracts $(100 \mu \mathrm{g} / \mathrm{mL})$ was added to a tube containing $1 \mathrm{~mL}$ of double-distilled water. Next, $0.075 \mathrm{~mL}$ of $5 \% \mathrm{NaNO}_{2}, 0.075 \mathrm{~mL}$ of $10 \% \mathrm{AlCl}_{3}$, and $0.5 \mathrm{~mL}$ of $1 \mathrm{M} \mathrm{NaOH}$ were added sequentially at 0,5 , and $6 \mathrm{~min}$. Finally, the volume of the reacting solution was adjusted to $2.5 \mathrm{~mL}$ with double-distilled water. The solution had an absorbance of $410 \mathrm{~nm}$, which was detected using an Ultrospec 2100 Pro Spectrophotometer (Section 3.3). The results were expressed in mg quercetin equivalents $(\mathrm{QE}) / \mathrm{g}$. 


\subsection{Measurement of Free Radical Scavenging Activity Using the 2,2-Diphenyl-1-picrylhydrazyl}

(DPPH) Assay

The free radical scavenging activity of SBS $(100 \mu \mathrm{g} / \mathrm{mL}$ in DW) was measured using the method of Brand-Williams [21] with some modification. L-Ascorbic acid was used as a positive control. The inhibition percentage was calculated from the following equation:

Inhibition $\%=[($ absorbance of control-absorbance of sample $) /$ absorbance of control $] \times 100$

The absorbance was measured using a spectrophotometer (Ultrospec 2100 pro; Amersham Pharmacia Biotech Co., Piscataway, NJ, USA).

\subsection{Measurement of Hydroxyl (OH') Radical Scavenging Activity}

Scavenging activity of SBS extracts on the hydroxyl radical $\left(\mathrm{OH}^{-}\right)$was measured using the deoxyribose method [22] with a slight modification. The deoxyribose assay was performed in $10 \mathrm{mM}$ phosphate buffer ( $\mathrm{pH}$ 7.4) containing $2.5 \mathrm{mM}$ deoxyribose, $1.5 \mathrm{mM} \mathrm{H}_{2} \mathrm{O}_{2}, 100 \mu \mathrm{M} \mathrm{FeCl}$, $104 \mu \mathrm{M}$ EDTA, and the extracts $(1 \mathrm{mg} / \mathrm{mL})$. The reaction was started by addition of ascorbic acid to the final concentration of $100 \mu \mathrm{M}$. The reaction mixture was incubated at $37{ }^{\circ} \mathrm{C}$ for $1 \mathrm{~h}$ in a water-bath and after incubation, the color was developed by addition of $0.5 \%$ thiobarbituric acid followed by ice-cold $2.8 \%$ trichloroacetic acid in $25 \mathrm{mM} \mathrm{NaOH}$ and heated at $80{ }^{\circ} \mathrm{C}$ for $30 \mathrm{~min}$. The extracts $(A 2)$ were cooled on ice and the absorbance was measured at $532 \mathrm{~nm}$. The reaction mixture without the test sample was used as a control $(A 1)$. The hydroxyl radical scavenging activity (HRSA) was calculated using the following equation:

$$
\operatorname{HRSA} \%=(A 1-A 2 / A 1) \times 100
$$

The inhibition curve was plotted for four experiments and was expressed as the $\%$ of the mean inhibition \pm SD.

\subsection{Measurement of ABTS Radical Scavenging Activity}

ABTS radical scavenging activity of SBS extracts and fractions was measured using the ABTS cation decolorization assay, as previously described [23], with some modifications. The ABTS radical cation $\left(\mathrm{ABTS}^{\circ+}\right.$ ) was produced by reaction of $7 \mathrm{mM}$ stock solution of ABTS with $2.45 \mathrm{mM}$ potassium persulfate and allowing the mixture to stand in the dark at room temperature for $12 \mathrm{~h}$ before use. The $\mathrm{ABTS}^{\circ+}$ solution was diluted with methanol to give an absorbance of $0.7 \pm 0.01$ at $734 \mathrm{~nm}$. Plant extracts and fractions $(1 \mathrm{~mL})$ were allowed to react with $2 \mathrm{~mL}$ of the $\mathrm{ABTS}^{\circ+}$ solution and the absorbance was measured at $734 \mathrm{~nm}$ after $1 \mathrm{~min}$. Trolox was used as a reference compound. The results were expressed as Trolox equivalent antioxidant capacity (TEAC) values and calculated as mean value $\pm \operatorname{SD}(n=4)$.

\subsection{High-Performance Liquid Chromatography (HPLC) Analysis of SBS Compounds}

Analysis of the compounds in the extract was performed using an Agilent 1100 series HPLC unit, equipped with a DAD. Samples were separated on a Nucleosil 100-5 C-18 column $(250 \mathrm{~mm} \times 4.0 \mathrm{~mm}$, 
i.d., $5 \mu \mathrm{m}$ particle size) which was protected by a $10 \mathrm{~mm}$ guard column with a gradient elution system. The mobile phase consisted of two solvents: solvent A was a mixture of water/formic acid ( $\mathrm{pH} 3.29)$, and solvent $\mathrm{B}$ was $100 \%$ acetonitril/formic acid ( $\mathrm{pH}$ 3.29). Gradient elution was performed as follows: initially, $7.0 \%$ of solvent B, followed by $0 \%$ to $15 \% \mathrm{~B}$ in $25 \mathrm{~min}, 30 \% \mathrm{~B}$ at $35 \mathrm{~min}, 40 \% \mathrm{~B}$ at $50 \mathrm{~min}$, $100 \% \mathrm{~B}$ at $45 \mathrm{~min}$, and $100 \% \mathrm{~B}$ at $55 \mathrm{~min}$. The flow rate was $1.0 \mathrm{~mL} / \mathrm{min}$, and the column temperature was $30{ }^{\circ} \mathrm{C}$. Used phenolic or flavonoid standard were performed by comparing retention times. The injections of sample and standard were performed in triplicate.

\subsection{Animals and Diets}

Four-week-old male Sprague-Dawley (Central Lab. Animal Inc.) rats were purchased from Central Lab. Animal Inc. (Seoul, Korea). Rats were acclimatized to the experimental facility for one week prior to the start of the study. The rats were divided into three groups of 10 and housed individually in polycarbonate cages in a room maintained at $22{ }^{\circ} \mathrm{C}$ and $55 \%$ relative humidity. The room was exposed to alternating $12 \mathrm{~h}$ periods of light and dark. All of the rats were allowed free access to food and water for five weeks. Food intake was measured daily, and the rats were weighed twice per week. After one week of acclimatization, rats were randomly divided into three groups: a normal diet group (ND, $n=10)$, a high-fat diet group (HFD, $n=10$ ), and a SBS group (HFD + SBS $150 \mathrm{mg} / \mathrm{kg} \mathrm{BW,} n=10$ ). Rats in the ND group were fed a normal diet (\#55VXT0038, Samyang Co, Korea). Obese rats were generated by feeding rats a high-fat diet, and rats in the HFD groups were fed a HFD based on a commercial diet (rodent diet with $60 \% \mathrm{kcal}$ fat, Research Diet, Korea). The composition of the diets and energy densities were presented in Table 1. The study protocol was approved by the Animal Care and Use Committee of Gyeongsang National University (Approval Number: GNU-130525-R0042).

Table 1. Ingredient composition of the experimental diet.

\begin{tabular}{ccc}
\hline Ingredient (milligram) & HFD & HFD + SBS (mg/kg/day) \\
\hline Casein & 200 & 200 \\
L-Cystine & 3 & 3 \\
Maltodextrin 10 & 125 & 125 \\
Sucrose & 68.8 & 68.8 \\
Cellulose & 50 & 50 \\
Soybean Oil & 25 & 25 \\
Lard & 245 & 245 \\
Mineral Mix S10026 & 10 & 10 \\
DiCalcium Phosphate & 13 & 13 \\
Calcium Carbonate & 5.5 & 5.5 \\
Potassuium Citrate & 16.5 & 16.5 \\
Vitamin Mix V10001 & 10 & 10 \\
Choline Bitartrate & 2 & 2 \\
Protein (milligram\%) & 26.2 & 26.2 \\
Carbohydrate (milligram\%) & 26.3 & 26.3 \\
Fat (milligram\%) & 34.9 & 34.9 \\
SBS extracts & - & 150 \\
\hline
\end{tabular}




\subsection{Biochemical Analysis}

Biochemical analysis was performed using commercial kits. Blood was placed in tubes containing $\mathrm{EDTA}_{2} \mathrm{Na}$, and serum was obtained by centrifuging the blood at $3000 \times \mathrm{g} 10 \mathrm{~min}$ at $4{ }^{\circ} \mathrm{C}$. Serum levels of aspartate aminotransferase (AST) and alanine aminotransferase (ALT) were detected using a Dry-chem Chemistry analyzer (Fujifilm, Japan). Excessive accumulation of triglyceride (TG) in the liver is the hallmark of NAFLD. Serum TG levels were assayed enzymatically using commercial kits (Asan phams, Co., Korea). For determination of hepatic TG content, $250 \mathrm{mg}$ of liver was homogenized in $4 \mathrm{~mL}$ of chloroform/methanol $(2: 1, \mathrm{v} / \mathrm{v})$, and $1 \mathrm{~mL}$ of $50 \mathrm{mM} \mathrm{NaCl}$ was added to each sample. The samples were then centrifuged, and the organic layer was removed and dried. The resulting pellet was dissolved in phosphate-buffered saline containing $1 \%$ Triton X-100, and the triglyceride content was determined using a commercially available enzymatic reagent kit (Asan phams, Co., Korea). The concentrations of total-cholesterol (TC) and high-density lipoprotein (HDL)-cholesterol were assayed enzymatically using commercial kits (Asan phams, Co., Korea).

\subsection{Histopathological Examinations}

Liver samples obtained at $12 \mathrm{~h}$ after the last administration of SBS extracts were fixed in $4 \%$ phosphate-buffered 4\% Paraformaldehyde, then processed routinely, embedded in paraffin, and sectioned to $5 \mu \mathrm{m}$ thickness. The sections were deparaffinized, rehydrated using standard techniques, stained with hematoxylin and eosin (H and $\mathrm{E}$ ), and examined by light microscopy.

\subsection{Gene Expression Analysis}

Total RNA was isolated from liver tissues from the different rat groups using Trizol reagent (Invitrogen, CA, USA). One microgram of total RNA was subjected to first strand cDNA synthesis using oligo (deoxythymidine) primers and Superscript II reverse transcriptase (Invitrogen, CA, USA). The target cDNA was amplified using the following sense and antisense primers: sense 5'-GGA GCC ATGGATTGCACATT-3' and antisense 5'-AGGAAGGCTTCCAGAGAGGA-3' for SREBP-1c; sense 5'-AAGGCTATCCCAGGCTTTGC-3' and antisense 5'-CGTCTGACTCGGTCTTCTTG-3' for PPAR $\alpha$; sense 5'-TTTTCAAGGGTGCCAGTTTC-3' and antisense 5'-AATCCTTGGCCCTCTGAGAT-3' for PPAR $\gamma$; sense 5'-TGCTAGAGGCCCTGCTACCAC-3' and antisense 5'-TGTGCACAGACACCTT CCCATC-3' for FAS; sense 5'-AGGAAGATGGTGTCC CGCTCTG-3' and antisense 5'-GGGG AGATGTGCTGGGTCAT-3' for ACC; sense 5'-TACAAGCAGGTGATCTTTGAGG-3' and antisense 5'-GGGCGAAACCAATATACTTCTG-3' for DGTA2. Control detection of $\beta$-actin was performed using sense 5'-AGGTCATCACTATCGGCAAT-3' and antisense 5'-ACTCATCGTACT CCTGCTTG- $3^{\prime}$ primers. PCR products were separated by electrophoresis on $1.5 \%$ agarose gel for $30 \mathrm{~min}$ at $100 \mathrm{~V}$. Gels were stained with $1 \mathrm{mg} / \mathrm{mL}$ ethidium bromide visualized by UV light using BIO-RAD Gel Doc image analysis software (BIO-RAD Laboratories Inc., CA, USA). All PCR products measured were normalized to the amount of $\beta$-actin cDNA in each sample. The mPNA levels are expressed as a ratio relative to $\beta$-actin mRNA. 


\subsection{Statistical Analysis}

Data are expressed as mean \pm SD. Significant differences among the treatment means were determined using ANOVA followed by Tukey's multiple comparisons test. $p<0.05$ was considered statistically significant.

\section{Results}

\subsection{Total Phenol Content (TPC) and Total Flavonoid Content (TFC) of SBS Extracts}

Data from analysis of total phenolic and flavonoid contents in the methanol extract from SBS are shown in Table 2. The phenolic contents of the extracts are shown as (+)-catechin equivalents (CQ) and flavonoids as quercetin equivalents (QE). The total phenolic content was $430.3 \mathrm{mg} \mathrm{CE} / \mathrm{g}$ dry weight and the total flavonoid content of SBS extracts was $127.5 \mathrm{mg}$ QE/g dry weight, respectively (Table 2).

Table 2. Antioxidant capacities, total phenolic and flavonoids content of Sasa borealis stem (SBS) extracts.

\begin{tabular}{|c|c|c|c|c|c|}
\hline & \multirow{2}{*}{$\begin{array}{c}\text { TPC } \\
\text { (mg Gallic acid/g } \\
\text { extract) }\end{array}$} & \multirow{2}{*}{$\begin{array}{c}\text { TFC } \\
\text { (mg Quercetin/g extract) }\end{array}$} & \multicolumn{2}{|c|}{ IC50 $(\mu \mathrm{g} / \mathrm{mL})$} & \multirow[b]{2}{*}{$\begin{array}{l}\text { ABTS assay } \\
\text { TEAC }\end{array}$} \\
\hline & & & $\begin{array}{c}\text { DPPH } \\
\text { scavenging assay }\end{array}$ & $\begin{array}{c}\text { HRSA } \\
\text { scavenging assay }\end{array}$ & \\
\hline SBS & $430.0 \pm 40.2$ & $127.5 \pm 12.0$ & $43.7 \pm 2.3$ & $450.5 \pm 28.5$ & $0.6 \pm 0.05$ \\
\hline
\end{tabular}

\subsection{Antioxidant Activity of SBS Extracts}

The antioxidant activities of SBS extracts were determined using the DPPH, ABTS, and HRSA assays. Data on DPPH radical scavenging activity of SBS are shown in Table 2. The results were expressed as $\mathrm{IC}_{50}$, which indicates the antioxidant concentration necessary for scavenging the initial DPPH concentration by $50 \%$. The SBS extract also inhibited hydroxyl radical generation and was found to possess strong antioxidant activity in hydroxyl radical scavenging activity. Trolox equivalent antioxidant capacity (TEAC) assay is one of the most commonly employed methods for determining antioxidant capacity. The TEAC assay measures the ability of a compound to scavenge ABTS radicals, and is widely used in screening of antioxidant activity of fruits, vegetables, and plants. The result of antioxidant activity of SBS extracts was expressed as TEAC values, as shown in Table 2. SBS showed high radical scavenging potential.

TPC, total phenolic content; TPC, total flavonoid content. Data are presented as the mean \pm SD $(n=4)$. TPC expressed as milligrams of gallic acid per gram of dry weight. TFC expressed as milligrams of Quercertin equivalent per gram of dry weight. Scavenging of free radicals by SBS extracts according to DPPH, Hydroxyl $\left(\mathrm{OH}^{-}\right)$, and ABTS scavenging assay. DPPH, DPPH radical scavenging activity; ABST, ABST radical scavenging activity; HRSA, hydroxyl radial scavenging activity. Data are presented as mean $\pm \mathrm{SD}(n=4)$. The antioxidant activity was evaluated as the concentration of tested sample required to scavenge $50 \%$ of the DPPH and HRSA. Trolox equivalent antioxidant capacity (TEAC) assay measured the ability of SBS to scavenge ABTS radicals. SBS extracts also contained phloroglucinol, 4-hydroxy benzhydrazide, garlic acid, vanillic acid, caffeic acid, syringic acid, chlorogenic acid, p-coumaric acid, trans-ferulic acid, sinapic acid, 2-amino-3, 
4-dimethyl-benzoic acid, protocatechuic acid, and coumarin. Among phenolic compounds, protocatechuic acid, coumarin, and $p$-coumaric acid were the main materials. In addition, SBS extracts also had many flavonoids, such as gallocatechin, epigallocatechin, catechin hydrate, epicatechin, epigallocatechin gallate, rutin hydrate, naringin, quercetin hydrate, myricetin, quercetin dehydrate, luteolin, and kaempferol (Table 3).

Table 3. Concentrations of phenolic acids and flavonoids of SBS extracts.

\begin{tabular}{|c|c|c|c|c|c|}
\hline Standards & RT $^{1}$ & $\lambda^{2}$ & Calibration curve & $\mathrm{LOQ}^{3}$ & Compounds \\
\hline \multicolumn{6}{|l|}{ Phenolic acids } \\
\hline Phloroglucinol & 7.27 & 280 & $Y=397.949 X+0.655$ & 50.00 & $0.60 \pm 0.00$ \\
\hline 4-Hydroxy benzhydrazide derivative & 7.57 & 280 & $Y=8119.555 X-59.083$ & 50.00 & $0.26 \pm 0.00$ \\
\hline Gallic acid & 8.74 & 280 & $Y=18,200.182 X-28.003$ & 50.00 & $0.53 \pm 0.00$ \\
\hline Vanillic acid & 21.91 & 280 & $Y=11,026.185 X+14.026$ & 50.00 & $1.44 \pm 0.01$ \\
\hline Caffeic acid & 22.21 & 280 & $Y=19,697.774 X-13.018$ & 50.00 & $0.74 \pm 0.00$ \\
\hline Syringic acid & 24.10 & 280 & $Y=17,500.224 X-1.523$ & 5.00 & $1.41 \pm 0.01$ \\
\hline Chlorogenic acid & 24.92 & 280 & $Y=6240.064 X-10.524$ & 50.00 & $1.61 \pm 0.01$ \\
\hline$p$-Coumaric acid & 32.87 & 280 & $Y=23,926.358 X+0.631$ & 5.00 & $10.41 \pm 0.05$ \\
\hline trans-Ferulic acid & 34.48 & 280 & $Y=16,058.167 X-17.063$ & 50.00 & $3.44 \pm 0.02$ \\
\hline Sinapic acid & 34.91 & 280 & $Y=7025.930 X+0.785$ & 50.00 & $2.98 \pm 0.01$ \\
\hline 2-Amino-3,4-dimethyl-benzoicacid & 35.30 & 280 & $Y=1209.000 X+0.000$ & 50.00 & $6.95 \pm 0.04$ \\
\hline$p$-Anisic acid & 35.40 & 280 & $Y=9558.576 X+5.493$ & 50.00 & - \\
\hline Protocatechuic acid ethyl ester & 36.89 & 280 & $Y=8796.340 X-2.765$ & 50.00 & $13.05 \pm 0.06$ \\
\hline Coumarin & 38.27 & 280 & $Y=24,055.754 X+48.641$ & 4.00 & $14.91 \pm 0.04$ \\
\hline $\mathrm{DPBA}^{4}$ & 39.85 & 280 & $Y=2971.415 X-7.872$ & 50.00 & - \\
\hline Alizarin & 43.86 & 280 & $Y=15,428.805 X+19.936$ & 1.00 & - \\
\hline Total phenolic acids & & & & & $62.98 \pm 0.08$ \\
\hline \multicolumn{6}{|l|}{ Flavonoids } \\
\hline Gallocatechin & 17.68 & 280 & $Y=1331.637 X+0.000$ & 50.00 & $1.59 \pm 0.00$ \\
\hline Epigallocatechin & 18.58 & 280 & $Y=96.137 X-0.550$ & 50.00 & $0.73 \pm 0.00$ \\
\hline Catechin hydrate & 23.66 & 280 & $Y=3982.083 X-6.943$ & 5.00 & $2.65 \pm 0.01$ \\
\hline Epicatechin & 28.00 & 280 & $Y=7641.670 X-14.487$ & 50.00 & $2.47 \pm 0.00$ \\
\hline Epigallocatechin gallate & 29.53 & 280 & $Y=6425.894 X-6.592$ & 50.00 & $0.98 \pm 0.00$ \\
\hline Rutin hydrate & 32.93 & 370 & $Y=4763.242 X-4.752$ & 50.00 & $0.29 \pm 0.01$ \\
\hline Catechin gallate & 33.77 & 280 & $Y=1462.905 X-1.970$ & 50.00 & - \\
\hline Naringin & 34.14 & 280 & $Y=8230.457 X-42.997$ & 50.00 & $2.60 \pm 0.02$ \\
\hline Quercetin hydrate & 37.53 & 370 & $Y=7476.858 X-6.972$ & 50.00 & $2.48 \pm 0.02$ \\
\hline Myricetin & 37.41 & 370 & $Y=9908.955 X-0.383$ & 5.00 & $0.43 \pm 0.00$ \\
\hline Morin hydrate & 38.48 & 320 & $Y=4100.693 X-4.129$ & 50.00 & - \\
\hline Quercetin dehydrate & 40.19 & 370 & $Y=5623.574 X-0.729$ & 50.00 & $1.93 \pm 0.00$ \\
\hline Luteolin & 40.28 & 370 & $Y=12,303.249 X-8.820$ & 50.00 & $6.32 \pm 0.03$ \\
\hline Kaempferol & 42.89 & 370 & $Y=12,894.258 X+38.962$ & 20.00 & $0.95 \pm 0.03$ \\
\hline 3-Hydroxyflavone & 45.75 & 320 & $Y=4687.303 X+0.191$ & 50.00 & - \\
\hline Total flavonoids & & & & & $23.42 \pm 0.09$ \\
\hline
\end{tabular}

Table 3 expressed by the RT, $\lambda$, calibration curve, LOQ and detected compounds. ${ }^{1}$ RT, retention time; ${ }^{2} \lambda$, absorbance (nm); ${ }^{3}$ LOQ, limit of quantitation; ${ }^{4}$ DPBA, diphenylboric acid 2-aminoethyl ester. Data are presented as mean $\pm \mathrm{SD}(n=3)$. 


\subsection{Effect of SBS on Body Weight and Liver Weight in HFD-Fed Rats}

Body weight gain and food intake of animals fed the experimental diets are shown in Table 4 . No significant difference in food intake was observed among the groups during the experimental diet period. Consumption of HFD (Table 1) for five weeks resulted in significantly increased body weight compared to the normal diet (ND). In contrast, rats fed a HFD supplemented with SBS extracts had $30.5 \%$ lower body weights than rats fed a HFD alone. The mean liver weight in the HFD group was significantly increased compared to that of the ND group. However, the increases in mean liver weight in the HFD + SBS group were smaller than those in the HFD group (Table 4). Although administration of HFD was found to result in significant elevation of the liver weight of the HFD group compared to the ND group, treatment with SBS was found to result in strong attenuation of liver weight.

Table 4. Effects of SBS on body and liver weights in high-fat diet (HFD)-fed rats.

\begin{tabular}{cccc}
\hline & ND & HFD & HFD + SBS \\
\hline Food intake (g/day) & $12.03 \pm 1.1$ & $11.04 \pm 1.6$ & $11.44 \pm 1.5$ \\
\hline Body weight & & & \\
Initial weight $(\mathrm{g})$ & $136.2 \pm 4.3$ & $135.5 \pm 2.7$ & $134.2 \pm 3.7$ \\
Final weight $(\mathrm{g})$ & $338.7 \pm 16.8$ & $447.3 \pm 20.5^{*}$ & $388.5 \pm 15.5^{\#}$ \\
Weight gain (g/5 weeks) & $202.5 \pm 9.3$ & $311.8 \pm 16.3^{*}$ & $254.3 \pm 10.7^{\#}$ \\
Liver weight (g/5 weeks) & $2.5 \pm 0.2$ & $3.8 \pm 0.3^{*}$ & $3.2 \pm 0.3^{\#}$ \\
\hline
\end{tabular}

Rats fed a HFD were treated orally with SBS extracts at a dose of $150 \mathrm{mg} / \mathrm{kg}$ body weight. ND: normal diet group, HFD: High-fat diet group, HFD + SBS: High-fat diet plus SBS (150 mg/kg BW) group. Body weight was measured twice per week. The weight of the liver was calculated by dividing the liver tissue weight by body weight (liver tissue/body weight $\times 100)$. The values are expressed as the mean $\pm \mathrm{SD}(n=10)$. ${ }^{*} p<0.01$ compared to ND. ${ }^{\#} p<0.05$ compared to HFD.

\subsection{Effect of SBS on Serum Total Cholesterols and Triglyceride Levels}

To determine hepatic steatosis-preventing the effect of SBS on HFD-fed rats, the serum lipid levels were measured. The HFD group showed significantly elevated serum TG concentrations compared to the ND group (Figure 1A). Administration of SBS resulted in decreased serum TG by $35 \%$ compared with the HFD group (Figure 1A). In addition, we found that the TG levels in the SBS group were close to those in the ND group. The HFD group also showed significantly increased levels of TC relative to the ND group, while SBS caused a marked decrease in serum TC levels when compared to the HFD group. The serum HDL-cholesterol level was significantly increased in the HFD + SBS group, when compared with HFD alone (Figure 1A). For evaluation of liver function, ALT and AST activities were examined between groups. Significantly increased ALT levels were observed in the HFD group compared to the ND group, while treatment with SBS resulted in a marked decrease in the ALT level compared to the HFD group (Figure 1B). AST levels in rats supplemented with SBS were significantly lowered compared to those in the HFD group (Figure 1B). 
Figure. 1. Effect of SBS on serum lipid contents in HFD-fed rats. (A) Serum TG and TC levels in rats fed a normal diet (ND), high-fat diet (HFD), and high-fat diet supplemented with SBS. Serum TG and TC levels were significantly reduced in rats treated with HFD + SBS compared to HFD. The values are expressed as the mean $\pm \mathrm{SD}$. ${ }^{*} p<0.05,{ }^{* *} p<0.01$.

(B) Serum levels of AST and ALT of rats fed ND, HFD, and HFD + SBS. The values are expressed as the mean $\pm \mathrm{SD}$. $* p<0.05$, ** $p<0.01$.

(A)
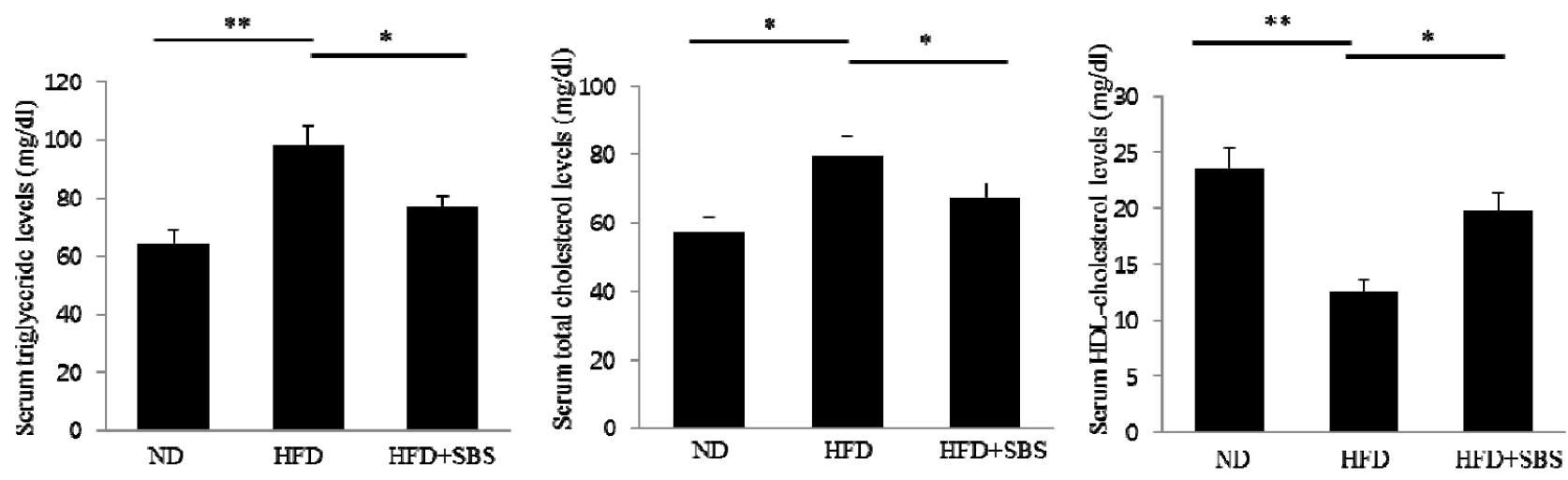

(B)
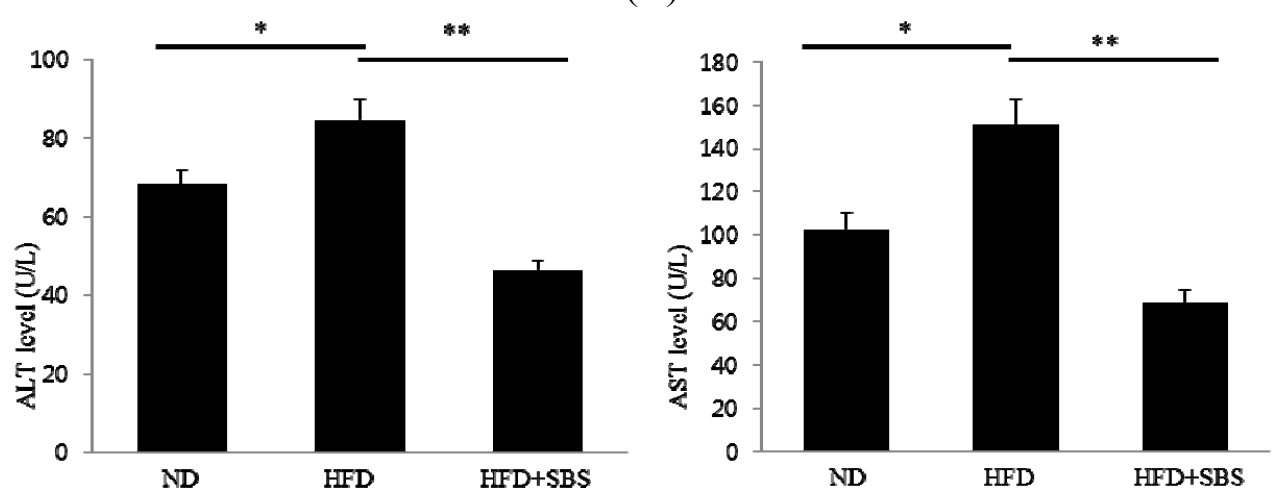

\subsection{Effects of SBS on Hepatic Total Cholesterols Levels and Triglyceride Levels}

To examine the effect of SBS on biochemical changes, we determined the levels of TG and TC in the liver. Administration of a HFD was found to result in significantly increased hepatic TG and TC in the HFD group. The SBS group, treated with SBS, showed markedly lower levels of TG and TC in the liver (Figure 2A). In addition, after a HFD for five weeks, the hepatic HDL-cholesterol levels showed a decrease compared with the ND group. However, the hepatic HDL-cholesterol levels in the SBS group increased by approximately $40 \%$ compared with the levels from rats on a HFD (Figure 2A). These results can be attributed to the ability of SBS to effectively suppress accumulation of hepatic TG and TC in HFD-fed rats.

\subsection{Effects of SBS on Hapatic Steatosis}

Next, $\mathrm{H}$ and $\mathrm{E}$ staining was performed for analysis of the effect of SBS on HFD-induced lipid accumulation in the liver. Lipid accumulation was highly amplified in the HFD group compared to the ND group. Histological examination of livers also revealed significant hepatic steatosis-characterized 
by swelling of hepatocytes and fat accumulation - in rats fed a HFD as compared with the HFD + SBS groups (Figure 2B). However, the lipid accumulations induced by HFD were significantly reduced in the HFD + SBS group, indicating that SBS is capable of preventing lipid accumulation and hepatic steatosis in HFD-induced fatty liver.

Figure 2. SBS reduces hepatic lipid levels in HFD-induced hepatic steatosis in rats. (A) Hepatic TG and TC levels in rats fed a normal diet (ND), high-fat diet (HFD), and high-fat diet supplemented with SBS. Significant decreases in the levels of hepatic triglyceride and total cholesterol were observed in the SBS-treated groups compared with HFD-induced obese rats. Values are expressed as mean $\pm \mathrm{SD}$. ${ }^{*} p<0.05,{ }^{*} p<0.01$. (B) Representative histological section of H \& E staining of liver prepared from rats fed ND, HFD, and HFD supplemented with SBS (magnification $200 \times$, scale bar $=100 \mu \mathrm{m}$ ). The major histological change induced by HFD in rat liver was hepatocyte steatosis and ballooning.
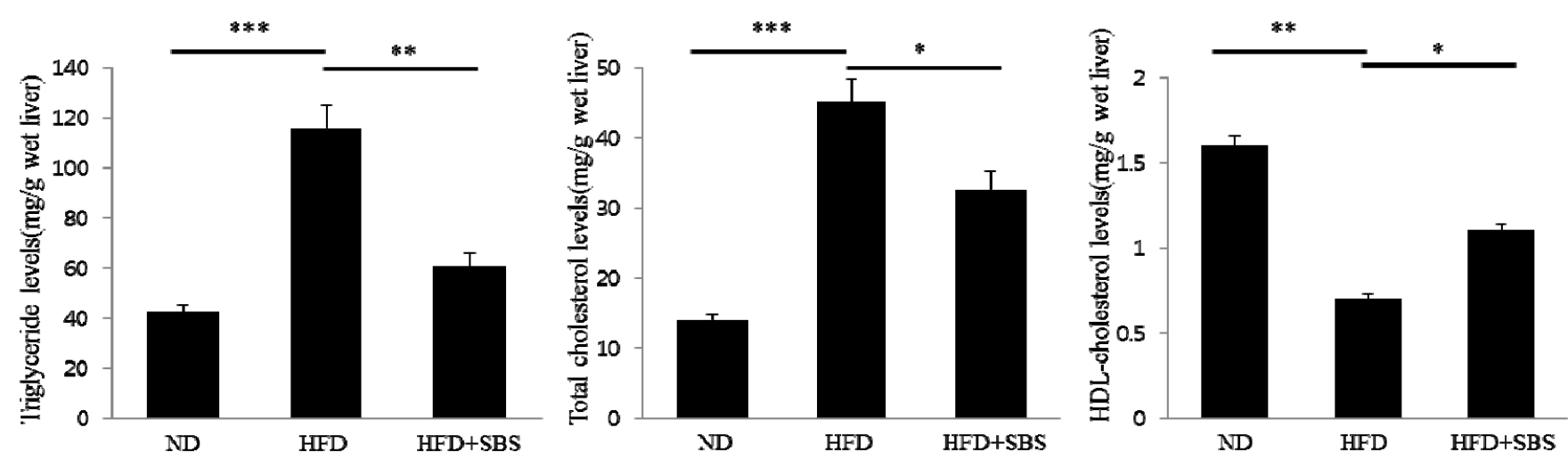

(B)

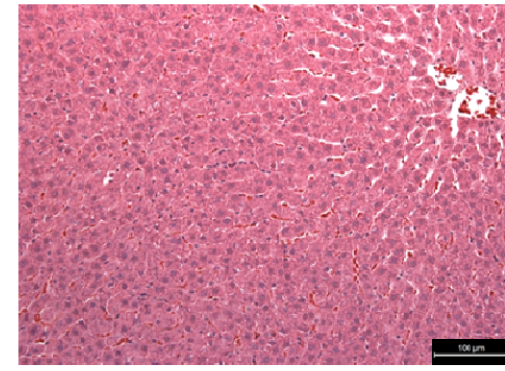

ND

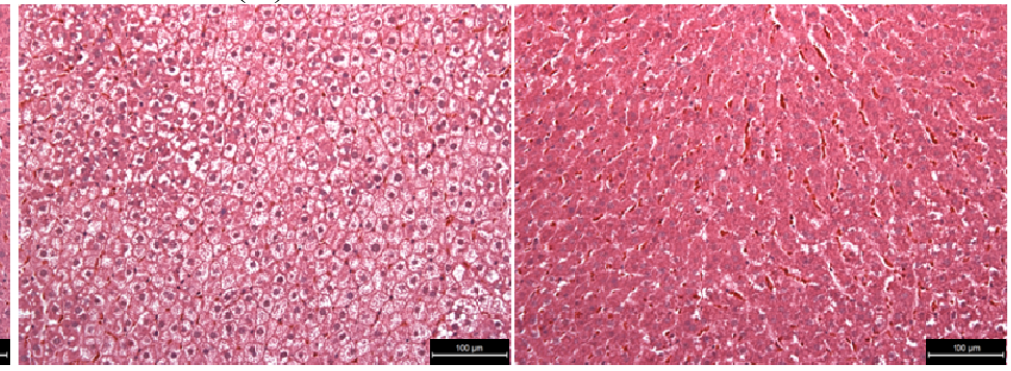

HFD
HFD+SBS

\subsection{Effects of SBS on Hepatic mRNA Levels of Lipid-Related Gene Expression}

For evaluation of the molecular events underlying the effects of SBS, we analyzed the expression of genes involved in lipid homeostasis in the liver. We assessed hepatic levels of lipogenesis-related genes (SREBP-1c, PPAR $\gamma$, and FAS), as well as fatty acid metabolism-related genes (PPAR $\alpha$, ACC, and DGAT2) in HFD-fed rats. According to our results, significantly lower gene expression levels of SREBP-1c, PPAR $\gamma$, and FAS, which promote synthesis of de novo monounsaturated fatty acid, were observed in the SBS-treated group than in the HFD group (Figure 3). Of these genes, expression of SREBP-1c and PPAR $\gamma$ showed the most significant reduction in the SBS group, compared to the HFD 
group (Figure 3). Hepatic mRNAs for PPAR $\alpha$ and ACC, the genes involved in fatty acid $\beta$-oxidation, were significantly decreased by BS administration when compared to the HFD group. In addition, SBS supplementation induced a significant decrease in the mRNA level of DGAT2 compared to the HFD group (Figure 3). Together, these results demonstrated that SBS contributed to inhibition of fatty acid synthesis, while activating fatty acid $\beta$-oxidation in livers of HFD-fed rats.

Figure 3. SBS inhibits expression of genes that regulate hepatic lipogenesis and fatty acid oxidation in hepatic steatosis. Total RNA isolated from liver was subjected to RT-PCR, and all of the gene transcripts were normalized using $\beta$-actin as a control. All of the experiments were performed in three independent experiments. Mean valves were significantly different from the ND group: $* p<0.05 ; * * p<0.01$; and $* * * p<0.001$. ND, rats fed a normal diet; HFD, rats fed a high-fat diet; HFD + SBS, rats fed a high-fat diet plus SBS (150 mg/kg BW); SREBP-1c, sterol regulatory element-binding protein-1c; PPAR a, peroxisome proliferator receptors-alpha; PPAR $\gamma$, peroxisome proliferator receptors-gamma; FAS, fatty-acid synthase; ACC, acetyl-CoA carboxylase; DGTA2, diacylglycerol acyltransferase 2 .
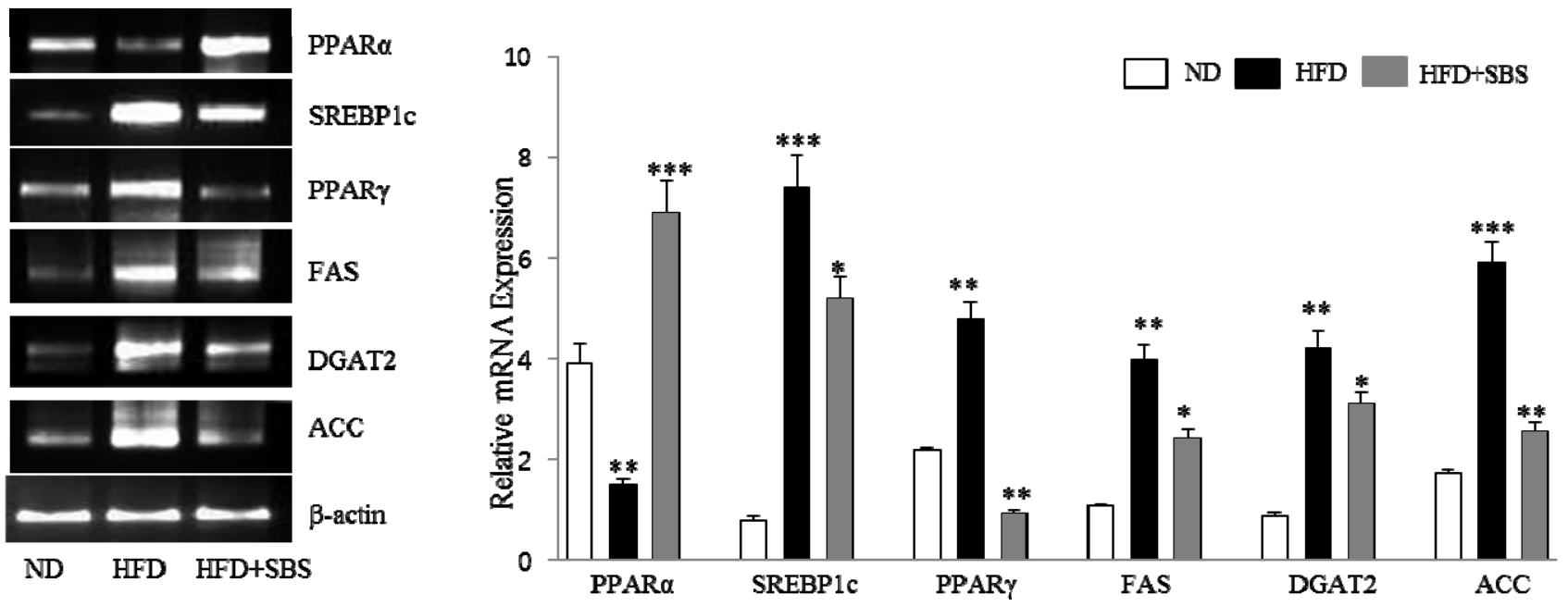

\section{Discussion}

Despite the rapidly growing recognition of hepatic steatosis over the past decade, therapy directed at treatment or prevention of the disease remains. Given the high prevalence of obesity in patients with hepatic steatosis, prevention of hepatic fat accumulation through weight reduction remains the cornerstone of treatment of hepatic steatosis [24]. In the current study, we investigated the anti-hepatic steatosis activity of SBS extract in HFD-fed rats. Our results demonstrated that, compared to the ND group, noticeably greater lipid accumulation in liver tissue and dramatically increased body and liver weights were observed in the HFD group. However, SBS supplementation in rats fed a HFD was effective in decreasing liver triglyceride and total cholesterol, and resulted in marked lowering of liver weight increases compared to the HFD group.

High fat diets cause weight gain, fat accumulation, and increased fat levels in the liver and serum. Fat accumulation in the liver increases the risks of NAFLD and non-alcoholic steatohepatitis (NASH), which cause hypercholesterolemia and cardiovascular disease [25]. Regarding the changes in lipid 
metabolism caused by fat accumulation, the aminotransferase activity of the liver is altered. Measurement of liver damage caused by fat accumulation in the liver is important for diagnosis of NAFLD. Therefore, AST and ALT were investigated as markers of liver damage. In the current study, levels of aspartate aminotransferase (AST) and alanine aminotransferase (ALT) showed a more significant increase in rats fed a HFD, compared to rats fed a ND. However, SBS supplementation resulted in significantly decreased levels of ALT and AST, demonstrating the robust hepatoprotective effects of SBS against HFD-induced liver damage.

Obesity causes altered function of adipocytes, which leads to expanded adipocyte mass and increased release of FFAs in the blood, which increases the amount of TG stored in the liver. Excess storage of TG in the liver results in significant and more abundant lipid accumulation, resulting in fatty liver [26,27]. In this study, rats in the HFD group showed a large number of lipid droplets and increased TG and cholesterol concentration in the liver and serum, confirming development of hepatic steatosis in the animal model. Administration of SBS to rats fed a HFD resulted in significantly decreased body weight as well as liver tissue weight. Treatment with SBS also resulted in a significant decrease in the hepatic and serum TG concentrations in HFD-induced hepatic steatosis in rats. In addition, our data from histopathological examination of livers from the rats showed a significant increase in the number and size of fatty hepatocytes upon HFD administration but returned to normal levels in rats that were administered SBS. These results demonstrated that the HFD-induced hepatic pathological changes were significantly inhibited in SBS-fed rats. These results clearly demonstrated that treatment with SBS resulted in effective improvement of hepatic steatosis induced by HFD.

The chemical compound cholesterol is a combination of lipid and steroid and is produced naturally by the body. Approximately $80 \%$ of the body's cholesterol is produced by and stored in the liver [28]. The liver is able to regulate cholesterol levels in the bloodstream and can secrete cholesterol if it is needed by the body. In the current study, we showed that administration of a HFD resulted in significantly increased levels of TC and decreased levels of HDL-cholesterol in serum and liver. However, administration of SBS resulted in decreased levels of serum and liver TC compared to the HFD group. In addition, SBS-fed rats showed liver HDL-cholesterol levels similar to those of the ND group.

Next, to explore the possible mechanism of SBS in decreasing accumulation of liver lipids, we investigated the expression levels of several genes related to fatty acid transport, and lipid metabolism, including lipogenesis and $\beta$-oxidation. Several studies have demonstrated the important role of SREBP-1c, a major transcription factor involved in hepatic lipogenesis, which leads to increases in fatty acid synthesis as a result of the induction of FAS and ACC [29,30]. One study reported that the level of SREBP-1c showed positive correlation with the degree of hepatic steatosis in patients with NAFLD [31]. Results of the current study showed that the level of SREBP-1c was significantly higher in the liver of rats fed a HFD compared with that of ND rats. However, SBS effectively inhibited the raise of SREBP-1c expression. FAS catalyzes the late step in fatty acid biosynthesis, thus, it is believed to be a major determinant of maximal hepatic capacity for generation of fatty acids by de novo lipogenesis. According to these results, administration of SBS resulted in decreased HFD-induced high expression of ACC and FAS, and expression of SREBP-1c transcriptional targets FAS and ACC showed strong correlation with SREBP-1c expression, suggesting that suppression of ACC and expression of FAS may contribute to a reduction in lipid accumulation in fatty liver. Together, since 
SBS supplementation in HFD rats resulted in significantly reduced serum and hepatic triglyceride, and cholesterol concentrations, these results demonstrated that SBS induced down-regulation of lipogenesis-related genes in HFD-induced fatty liver. Of particular importance, PPAR $\alpha$ is a ligand-activated transcription factor; its activation induces the mRNA expression of several genes involved in fatty acid oxidation to reduce the circulating lipid levels [32]. The current results showed significantly lower PPAR $\alpha$ expression in the HFD group than in the ND group, and it was increased by BS supplementation. Findings of a recent report indicated that PPAR $\alpha$ also modulates hepatic lipogenic gene expression, such as DGAT, ACC, and PGC- $1 \alpha$, which are closely related to fatty acid synthesis and oxidation in hepatic steatosis in HFD-fed animals [33]. DGAT is a microsomal enzyme that joins acyl-CoA to 1,2-diacylglycerol and thus constitutes the final step in TG biosynthesis [34]. Our results showed that DGAT2 expression in HFD rats was significantly increased, while DGAT2 mRNA expression showed a dramatic decrease in the SBS-treated group. Recent studies have suggested that overexpression of DGAT2 leads to increases in large cytosolic lipid droplets [34], and hepatic-specific overexpression of DGAT2 led to increased liver TG content [35]. Therefore, our results indicated that administration of SBS resulted in down-regulation of the levels of DGAT2 mRNA and reduction of DGAT2 expression in the HFD-SBS group inhibited accumulation of hepatic lipid droplets via a decrease of TG synthesis. In the current study, we also found that mRNA expression of PPAR $\gamma$ was significantly lower in the FHD-SBS group than in the HFD group, demonstrating that a SBS-supplemented diet induced a reduction of PPAR $\gamma$ expression in the HFD-induced steatosis liver. In addition, gene expression generally exhibits a negative relationship with the DNA methylation of $\mathrm{CpG}$ islands in the gene promoter region. Sookoian et al. [36] reported that an increase of PPAR- $\gamma$ coactivator $1 \alpha$ methylation correlated with decreased mRNA expression in the liver and contributed to insulin resistance in NAFLD patients. In HFD-fed mice, the increased methylation of the PPAR $\gamma$ promoter accompanied by the decreased expression of PPAR $\gamma$ mRNA in visceral adipose tissues was associated with the pathogenesis of metabolic syndrome [37]. Taken together, our results showed a significantly smaller liver lipid droplet area in the SBS group, accompanied by an increase in PPAR $\alpha$ and a decrease in expression of SREBP-1c, FAS, ACC, and DGAT2.

Medicinal plants or many natural products exhibiting good antioxidant activity are associated with hepatoprotection potential. Bamboo leaves exhibited significant antioxidant activity against the 1,1-diphenyl-2-picrylhydrazyl (DPPH) radical and a cytoprotective effect against oxidative damage in liver cells [17]. In the current study, we found that SBS extracts contained high phenolic contents (430 mg gallic acid equivalent/g extract) and flavonoid contents (127.5 mg quercetin equivalent/g extract), respectively. When we analyze SBS extracts using with HPLC-DAD, in terms of phenolic acids, SBS extracts had phloroglucinol, 4-hydroxy benzhydrazide, garlic acid, vanillic acid, caffeic acid, syringic acid, chlorogenic acid, $p$-coumaric acid, trans-ferulic acid, sinapic acid, 2-amino-3,4-dimethyl-benzoic acid, protocatechuic acid, and coumarin. Among phenolic compounds, protocatechuic acid, coumarin, and $p$-coumaric acid were the main materials. In addition, SBS extracts also had many flavonoids, such as gallocatechin, epigallocatechin, catechin hydrate, epicatechin, epigallocatechin gallate, rutin hydrate, naringin, quercetin hydrate, myricetin, quercetin dehydrate, luteolin, and kaempferol. Our results also showed that SBS had an effective capacity of scavenging for DPPH, ABTS, and hydroxyl radicals and showed correlation with potent phenol and flavonoid contents, thus, suggesting its antioxidant 
potential. The current finding suggested that hepatoprotective effects and anti-steatosis functions of SBS in HFD-induced NAFLD may be due to the potent antioxidant properties and to the abundant presence of phenolic and flavonoid contents of SBS.

\section{Conclusions}

In conclusion, these results indicated that SBS induced significant suppression of TG and cholesterol accumulation in the liver of rats fed a HFD. SBS possesses a repressive property on hepatic steatosis, which is associated with inhibition of SREBP1c, PPAR $\gamma$, ACC, DGAT2, and FAS, and induction of PPAR $\alpha$, suggesting a potential application of SBS in treatment of HFD-induced NAFLD.

\section{Acknowledgements}

The work was supported by iPET (112001032SB030); Ministry of Agriculture, Food and Rural Affairs and Basic Science Research Program of National Research Foundation (2013053810); Ministry of Education, Korea. This work was supported by Priority Research Centers Program through the National Research Foundation of Korea (NRF) funded by the Ministry of Education, Science and Technology (2009-0093813).

\section{Conflicts of Interest}

The authors declare no conflict of interest.

\section{References}

1. Ludwig, J.; Viggiano, T.R.; McGill, D.B.; Oh, B.J. Nonalcoholic steatohepatitis: Mayo clinic experience with a hitherto unnamed disease. Mayo Clin. Proc. 1980, 55, 434-438.

2. Marchesini, G.; Bugianesi, E.; Forlani, G.; Cerrelli, F.; Lenzi, M.; Manini, R.; Natale, S.; Vanni, E.; Villanova, N.; Melchionda, N.; Rizzetto, M. Nonalcoholic fatty liver, steatohepatitis, and the metabolic syndrome. Hepatology 2003, 37, 917-923.

3. Powell, E.E.; Cooksley, W.G.; Hanson, R.; Searle, J.; Halliday, J.W.; Powell, L.W. The natural history of nonalcoholic steatohepatitis: A follow-up study of forty-two patients for up to 21 years. Hepatology 1990, 11, 74-80.

4. Marchesini, G.; Brizi, M.; Bianchi, G.; Tomassetti, S.; Bugianesi, E.; Lenzi, M.; McCullough, A.J.; Natale, S.; Forlani, G.; Melchionda, N. Nonalcoholic fatty liver disease: A feature of the metabolic syndrome. Diabetes 2001, 50, 1844-1850.

5. Fon Tacer, K.; Rozman, D. Nonalcoholic fatty liver disease: Focus on lipoprotein and lipid deregulation. J. Lipids 2011, 2011, 783976.

6. Browning, J.D.; Horton, J.D. Molecular mediators of hepatic steatosis and liver injury. J. Clin. Investig. 2004, 114, 147-152.

7. Nagle, C.A.; Klett, E.L.; Coleman, R.A. Hepatic triacylglycerol accumulation and insulin resistance. J. Lipid Res. 2009, 50, S74-S79.

8. Prior, R.L.; Cao, G. Antioxidant capacity and polyphenolic components of teas: Implications for altering in vivo antioxidant status. Proc. Soc. Exp. Biol. Med. 1999, 220, 255-261. 
9. Lu, B.; Wu, X.; Tie, X.; Zhang, Y.; Zhang, Y. Toxicology and safety of anti-oxidant of bamboo leaves. Part 1: Acute and subchronic toxicity studies on anti-oxidant of bamboo leaves. Food Chem. Toxicol. 2005, 43, 783-792.

10. Lu, B.; Wu, X.; Shi, J.; Dong, Y.; Zhang, Y. Toxicology and safety of antioxidant of bamboo leaves. Part 2: Developmental toxicity test in rats with antioxidant of bamboo leaves. Food Chem. Toxicol. 2006, 44, 1739-1743.

11. Choi, Y.J.; Lim, H.S.; Choi, J.S.; Shin, S.Y.; Bae, J.Y.; Kang, S.W.; Kang, I.J.; Kang, Y.H. Blockade of chronic high glucose-induced endothelial apoptosis by Sasa borealis bamboo extract. Exp. Biol. Med. (Maywood) 2008, 233, 580-591.

12. Lee, M.J.; Park, W.H.; Song, Y.S.; Lee, Y.W.; Song, Y.O.; Moon, G.S. Effect of bamboo culm extract on oxidative stress and genetic expression: Bamboo culm extract ameliorates cell adhesion molecule expression and NFkappaB activity through the suppression of the oxidative stress. Clin. Nutr. 2008, 27, 755-763.

13. Park, E.J.; Jhon, D.Y. Effects of bamboo shoot consumption on lipid profiles and bowel function in healthy young women. Nutrition 2009, 25, 723-728.

14. Zhang, Y.; Yao, X.; Bao, B.; Zhang, Y. Anti-fatigue activity of a triterpenoid-rich extract from Chinese bamboo shavings (Caulis bamfusae in taeniam). Phytother. Res. 2006, 20, 872-876.

15. Ko, B.S.; Jun, D.W.; Jang, J.S.; Kim, J.H.; Park, S. Effect of Sasa borealis and white lotus root and leaves on insulin action and secretion in vitro. Korea J. Food Sci. Technol. 2006, 38, 114-120.

16. Kim, E.Y.; Jung, E.Y.; Lim, H.S.; Heo, Y.R. The effects of the Sasa borealis leaves extract on plasma adiponectin, restin, C-reactive protein and homocyteine levels in high fat diet-induced obese C57/BL6J mice. Korean J. Nutr. 2007, 40, 303-311.

17. Park, H.S.; Lim, J.H.; Kim, H.J.; Choi, H.J.; Lee, I.S. Antioxidant flavone glycosides from the leaves of Sasa borealis. Arch. Pharm. Res. 2007, 30, 161-166.

18. Shibata, M.; Yamatake, M.; Sakamoto, M.; Kanamori, K.; Takagi, K.; Okabe, S. Pharmacological studies on bamboo grass. Nippon Yakurigaku Zassi 1975, 71, 481-485.

19. Singleton, V.L.; Rossi, J.R. Colorimetry of total phenolics with phosphomolybdic-phosphotungstic acid. Am. J. Enol. Vitic. 1965, 16, 144-158.

20. Meda, A.; Lamien, C.E.; Romito, M.; Millogo, J.; Nacoulma, O.G. Determination of the total phenolic, flavonoid and proline contents in Burkina Fasan honey, as well as their radical scavenging activity. Food Chem. 2005, 91, 571-577.

21. Brand-Williams, W.; Cuvelier, M.E.; Berset, C. Use of a free radical method to evaluate antioxidant activity. LWT Food Sci. 1995, 28, 25-30.

22. Halliwell, B.; Gutteridge, J.M.C.; Arurma, O.I. The deoxyribose method: A simple "test-tube" assay for determination of rate constants for reactions of hydroxyl radicals. Anal. Biochem. 1987, $165,215-219$.

23. Re, R.; Pellegrini, N.; Proteggente, A.; Pannala, A.; Yang, M.; Rice-Evans, C. Antioxidant activity applying an improved ABTS radical cation decolorization assay. Free Radic. Biol. Med. 1999, 26, 1231-1237.

24. Beaton, M.D. Current treatment options for nonalcoholic fatty liver disease and nonalcoholic steatohepatitis. Can. J Gastroenterol. 2012, 26, 353-357. 
25. Hirako, S.; Kim, H.J.; Shimizu, S.; Chiba, H.; Matsumoto, A. Low-dose fish oil consumption prevents hepatic lipid accumulation in high cholesterol diet fed mice. J Agric. Food Chem. 2011, 59, 13353-13359.

26. Bradbury, M.W. Lipid metabolism and liver inflammation. I. Hepatic fatty acid uptake: Possible role in steatosis. Am. J. Physiol. Gastrointest. Liver Physiol. 2006, 290, G194-G198.

27. Cho, A.S.; Jeon, S.M.; Kim, M.J.; Yeo, J. Seo, K.I.; Choi, M.S.; Lee, M.K. Chlorogenic acid exhibits anti-obesity property and improves lipid metabolism in high-fat diet-induced-obese mice. Food Chem. Toxicol. 2010, 48, 937-943.

28. Kwon, H.J.; Kim, Y.Y.; Choung, S.Y. Amelioration effects of traditional Chinese medicine on alcohol-induced fatty liver. World J. Gastroenterol. 2005, 11, 5512-5516.

29. Sekiya, M.; Yahagi, N.; Matsuzaka, T.; Najima, Y.; Nakakuki, M.; Nagai, R.; Ishibashi, S.; Osuga, J.; Yamada, N.; Shimano, H. Polyunsaturated fatty acids ameliorate hepatic steatosis in obese mice by SREBP-1 suppression. Hepatology 2003, 38, 1529-1539.

30. Shimano, H.; Horton, J.D.; Shimomura, I.; Hammer, R.E.; Brown, M.S.; Goldstein, J.L. Isoform 1c of sterol regulatory element binding protein is less active than isoform 1a in livers of transgenic mice and in cultured cells. J. Clin. Investig. 1997, 99, 846-854.

31. Yang, Z.X.; Shen, W.; Sun, H. Effects of nuclear receptor FXR on the regulation of liver lipid metabolism in patients with non-alcoholic fatty liver disease. Hepatol. Int. 2010, 4, 741-748.

32. Takahashi, N.; Senda, M.; Lin, S.; Goto, T.; Yano, M.; Sasaki, T.; Murakami, S.; Kawada, T. Auraptene regulates gene expression involved in lipid metabolism through PPAR $\alpha$ activation in diabetic obese mice. Mol. Nutr. Food Res. 2011, 55, 1791-1797.

33. Nagasawa, T.; Inada, Y.; Nakano, S.; Tamura, T.; Takahashi, T.; Maruyama, K.; Yamazaki, Y.; Kuroda, J.; Shibata, N. Effects of bezafibrate, PPAR pan-agonist, and GW501516, PPARdelta agonist, on development of steatohepatitis in mice fed a methionine- and choline-deficient diet. Eur. J. Pharmacol. 2006, 536, 182-191.

34. Stone, S.J.; Myers, H.M.; Watkins, S.M.; Brown, B.E.; Feingold, K.R.; Elias, P.M.; Farese, R.V., Jr. Lipopenia and skin barrier abnormalities in DGAT2-deficient mice. J. Biol. Chem. 2004, 279, 11767-11776.

35. Yamazaki, T.; Sasaki, E.; Kakinuma, C.; Yano, T.; Miura, S.; Ezaki, O. Increased very low density lipoprotein secretion and gonadal fat mass in mice overexpressing liver DGAT. J. Biol. Chem. 2005, 280, 21506-21514.

36. Sookoian, S.; Rosselli, M.S.; Gemma, C.; Burgueño, A.L.; Fernández Gianotti, T.; Castaño, G.O.; Pirola, C.J. Epigenetic regulation of insulin resistance in nonalcoholic fatty liver disease: Impact of liver methylation of the peroxisome proliferator-activated receptor $\gamma$ coactivator $1 \alpha$ promoter. Hepatology 2010, 52, 1992-2000.

37. Fujiki, K.; Kano, F.; Shiota, K.; Murata, M. Expression of the peroxisome proliferator activated receptor gamma gene is repressed by DNA methylation in visceral adipose tissue of mouse models of diabetes. BMC Biol. 2009, 7, 38, doi:10.1186/1741-7007-7-38.

(C) 2014 by the authors; licensee MDPI, Basel, Switzerland. This article is an open access article distributed under the terms and conditions of the Creative Commons Attribution license (http://creativecommons.org/licenses/by/3.0/). 